\title{
Modelling the rotational curves of spiral galaxies with a scalar field
}

\author{
J. P. Mbelek
}

\author{
Service d'Astrophysique, C.E. Saclay, 91191 Gif-sur-Yvette Cedex, France \\ e-mail: mbelek@discovery.saclay.cea.fr
}

Received 6 June 2003 / Accepted 26 May 2004

\begin{abstract}
In a previous work (Mbelek 1998), we modelled the rotation curves (RC) of spiral galaxies by including in the equation of motion of the stars the dynamical terms from an external real self-interacting scalar field, $\psi$, minimally coupled to gravity and which respects the equivalence principle in the weak fields and low velocity approximation. This model appeared to have three free parameters: the turnover radius, $r_{0}$, the maximum tangential velocity, $v_{\theta \max }=v_{\theta}\left(r_{0}\right)$, plus a strictly positive integer, $n$. Here, we propose a new improved version where the coupling of the $\psi$-field to dark matter is emphasized at the expense of its self-interaction. This reformulation presents the very advantageous possibility that the same potential is used for all galaxies. Using at the same time a quasi-isothermal dark matter density and the scalar field helps to better fit the RC of spiral galaxies. In addition, new correlations are established.
\end{abstract}

Key words. cosmology: theory - cosmology: dark matter - galaxies: kinematics and dynamics

\section{Introduction}

Since 1998, many groups have been working on modelling the RC of spiral galaxies with ultra-light scalar fields as an alternative to the standard assumption of weakly-interacting massive particles. Guzman \& Matos (2000) presented a model where they assume that the galactic dark matter consists entirely of a self-interacting real scalar field $\Phi$ endowed with a potential $V(\Phi)$ which is an exponential function of $\Phi$. First they obtained an exact static and axially symmetric solution of the Einstein-dilaton theory (Guzman \& Matos 2000a). This solution yields an effective energy density $\mu_{\mathrm{DM}}=V(\Phi)$ which mimics an isothermal dark halo with a constant core density. The resulting circular velocity profile of test particles involves three free parameters to fit the observations. However, the authors (Guzman \& Matos 2000b) pointed out some difficulties related to the physical origin of the kind of exponential potential $V(\Phi)$ they have to consider. Based on a complex scalar field forming galactic halos after Bose condensation, Arbey et al. (2001a) have suggested a massive ( $m=0.4$ to $\left.1.6 \times 10^{-23} \mathrm{eV} / c^{2}\right)$ and non-interacting complex scalar field, as a dark matter candidate alternative to the neutralino, to bind galaxies and flatten the RC of spirals. Their motivation for a complex (i.e., charged) scalar field follows from the need for stable bounded configurations in the case of self-interacting bosons (not interacting with any other kind of matter). The model of the authors accounts better for the dark matter inside low-luminosity spirals than for the brightest objects where baryons dominate. However, because they deal with a charged scalar field (Arbey et al. 2001b), some problems (pointed out by the authors themselves) arise when the limits from galactic dynamics and some cosmological constraints are taken simultaneously into account.
Ureña-Lòpez \& Liddle (2002) have shown that the supermassive black holes hosted at the center of galaxies can coexist with scalar field halos. Since all the aforementioned studies consider only self-interacting scalar fields, the equation satisfied by their respective scalar field is of the sourceless Klein-Gordon type (including the derivative of the scalar field potential). The aim of this work is to show that a real scalar field, $\psi$, minimally coupled to gravity sourced by and interacting with matter (both baryonic and dark matter), also may reproduce the RC of spiral galaxies. This can be achieved if the force term involved by the scalar field can contribute sufficiently to lower the magnitude of the orbital momenta of stars and gas, even if $\psi$ itself does not contribute significantly to the mass of the dark halo. The general equation of $\psi$ reads (Mbelek \& Lachièze-Rey 2003)

$\nabla_{v} \nabla^{v} \psi=-J-\frac{\partial J}{\partial \psi} \psi-\frac{\partial U}{\partial \psi}$

where $U=U(\psi)$ and $J=J\left(x^{\alpha}\right)$ denote respectively the selfinteraction potential and the source term of the $\psi$-field; $\nabla_{v} \nabla^{v}$ is the d'Alembertian of the curved four dimensional spacetime endowed with the metric $\mathrm{d} s^{2}=g_{\mu \nu} \mathrm{d} x^{\mu} \mathrm{d} x^{\nu}$. The potential $U$ is of a symmetry breaking type but needs not to be specified here; $U\left(\psi_{\infty}\right)=0, \frac{\partial U}{\partial \psi}\left(\psi_{\infty}\right)=0$ and $\frac{\partial^{2} U}{\partial \psi^{2}}\left(\psi_{\infty}\right)>0$, where $\psi_{\infty}$ denotes the value of $\psi$ at the minimum of the potential $U(\psi)$. Equation (1) describes a minimally coupled classical real scalar field with the usual Lagrangian density (Narlikar \& Padmanabhan 1986)

$$
\mathcal{L}=\frac{c^{4}}{8 \pi G}\left[\frac{1}{2} \partial_{\mu} \psi \partial^{\mu} \psi-U-J \psi\right] \sqrt{-\operatorname{det}\left(g_{\mu \nu}\right)},
$$


but taking into account that the source term, $J$, may depend on the $\psi$-field too. This is the case when the coupling constants to the other form of matter-energy are generalized to be functions that depend also on the scalar field. For our purpose, one may neglect the contribution of the electromagnetic fields. Hence, $J$ accounts for the matter source only. As usual in the framework of scalar-tensor type theories, this is of the general form $J=\frac{4 \pi G}{c^{4}} g(\psi) T_{\alpha}^{\alpha}$ (see e.g., Straumann 2000), where $T_{\alpha}^{\alpha}$ is the trace of the energy-momentum tensor of the matter source and the coupling function $g$ is such that $g\left(\psi_{\infty}\right)=0$ in the vacuum state (minimum of the potential $U$ ).

\section{Weak and static field approximation}

Assuming that the excitation $\delta \psi=\psi-\psi_{\infty}$ of the $\psi$-field is small compared to $\psi_{\infty}$, the equation of the $\psi$-field reads in the first approximation for a static matter distribution

$\Delta \delta \psi-\lambda^{-2} \delta \psi=\frac{\partial g}{\partial \psi}\left(\psi_{\infty}\right) \psi_{\infty} \frac{4 \pi G}{c^{2}} \rho$,

where $\lambda=\left(\frac{\partial^{2} U}{\partial \psi^{2}}\left(\psi_{\infty}\right)\right)^{-1 / 2}$ denotes the range of the $\psi$-field and $\rho$ is the mass density of the matter fields other than the $\psi$-field itself. Out of the matter source, Eq. (3) reduces to

$\Delta \delta \psi-\lambda^{-2} \delta \psi=0$

whose solution is the familiar Yukawa potential $\delta \psi \propto$ $\exp (-\lambda r) / r$. Accordingly, $\psi \rightarrow \psi_{\infty}$ at infinity. Since $\psi$ is long range (say $\lambda \geq 100 \mathrm{kpc}$ ), we may neglect in the first approximation the scalar field mass term, $\lambda^{-2}$, with respect to the mass density terms within galaxies. Thus, Eq. (3) simplifies as follows

$\Delta \psi=\frac{\partial g}{\partial \psi}\left(\psi_{\infty}\right) \psi_{\infty} \frac{4 \pi G}{c^{2}} \rho$.

\section{The equation of motion}

The equation of motion of test bodies is obtained following the usual procedure (Ciufolini \& Wheeler 1995) which consists of considering a gas of dust (pressureless perfect fluid) whose energy-momentum tensor reads $T^{\mu \nu}=\rho c^{2} u^{\mu} u^{v}$, where the density $\rho$ is a constant. The equation of motion is then derived from the conservation of the energy-momentum tensor, that is $\nabla_{v} T^{\mu v}=0$ (where $\nabla_{v}$ denotes the covariant derivative $\nabla_{v} T^{\mu v}=\partial_{v} T^{\mu v}+\Gamma_{\sigma v}^{\sigma} T^{\mu v}+\Gamma_{\sigma v}^{\mu} T^{\sigma v}$, and $\Gamma_{\sigma v}^{\mu}=\frac{1}{2} g^{\mu \lambda}\left(\frac{\partial g_{\sigma \lambda}}{\partial x^{\nu}}+\right.$ $\left.\frac{\partial g_{\lambda v}}{\partial x^{\sigma}}-\frac{\partial g_{\sigma v}}{\partial x^{\lambda}}\right)$ defines the Christoffel symbols). In our framework, the same procedure applies to an appropriate effective energymomentum tensor $T_{\text {eff }}^{\mu v}$ which accounts for the presence of the scalar field. Since the trace $T_{\alpha}^{\alpha}$ is involved in the source term $J$, arranging in order to isolate all the contributions of the matter in the total Lagrangian density leads to the identification $T_{\text {eff }}^{\mu \nu}=T^{\mu \nu}+g(\psi) \psi T_{\alpha}^{\alpha} g^{\mu \nu}$. As a consequence, this does not lead to the geodesic equation since the influence of the scalar field now enters. Indeed, it follows for a neutral test body

$$
\frac{\mathrm{d} u^{\mu}}{\mathrm{d} s}+\Gamma_{\alpha \beta}^{\mu} u^{\alpha} u^{\beta}=\frac{\mathrm{d}(g(\psi) \psi)}{\mathrm{d} s} u^{\mu}-\partial^{\mu}(g(\psi) \psi)
$$

Note that Eq. (6) is generic in scalar-tensor theory like the Brans-Dicke (Brans \& Dicke 1961) theory or the 5D compactified Kaluza-Klein theory (Wesson \& Ponce de Leon 1995). Independently of the physical meaning, Eq. (6) may be obtained from the variational principle $0=\delta \int m c \sqrt{g_{\mu \nu} u^{\mu} u^{v}} \mathrm{~d} s$ with an effective mass $m \propto \exp (g(\psi) \psi)$. The geodesic equation is recovered in the case of a non variable $\psi$-field. Now, in the weak field and low velocity limit, one gets $\Gamma_{00}^{\mu} \approx-\frac{1}{2} g^{\mu \mu} \partial^{\mu} g_{00}$ (with no summation on $\mu$ ). Hence, in the first order approximation, Eq. (6) simplifies to

$$
\frac{\mathrm{d} \boldsymbol{v}}{\mathrm{d} t}=-\boldsymbol{\nabla}\left(V_{\mathrm{N}}+c^{2} \frac{\partial g}{\partial \psi}\left(\psi_{\infty}\right) \psi_{\infty} \psi\right)+\frac{\partial g}{\partial \psi}\left(\psi_{\infty}\right) \psi_{\infty} \frac{\mathrm{d} \psi}{\mathrm{d} t} \boldsymbol{v},
$$

where the Newtonian potential, $V_{\mathrm{N}}$, results from the contributions of the visible and dark matter components including that of the $\psi$-field. Newtonian physics is recovered in the limit case $\delta \psi \rightarrow 0$.

\section{Modelling the RC of spirals}

Since a drag-like force term appears in Eq. (7), the rotational velocity $v$ should read in the general form $v=\sqrt{v_{\theta}^{2}+\dot{r}^{2}}$, where the tangential velocity $v_{\theta}$ and the radial velocity $\dot{r}$ are derived in spherical coordinates respectively from the tangential equation

$\frac{1}{r} \frac{\mathrm{d}\left(r v_{\theta}\right)}{\mathrm{d} t}=\frac{\partial g}{\partial \psi}\left(\psi_{\infty}\right) \psi_{\infty} \frac{\mathrm{d} \psi}{\mathrm{d} t} v_{\theta}$

and the radial equation

$\ddot{r}-\frac{v_{\theta}^{2}}{r}=-G \frac{m(r)+m^{(\psi)}(r)}{r^{2}}$,

where we have set $m^{(\psi)}(r)=\frac{c^{2}}{G} \frac{\partial g}{\partial \psi}\left(\psi_{\infty}\right) \psi_{\infty} \frac{\mathrm{d} \psi}{\mathrm{d} r} r^{2}$ and $m(r)=$ $m_{\mathrm{vis}}(r)+m_{\mathrm{dark}}(r)$ is the total mass up to radius $r$ assuming spherical symmetry. In the latter relation, $m_{\mathrm{vis}}(r)$ denotes the visible matter mass and $m_{\mathrm{dark}}(r)$ the dark matter mass including both the contributions of the dark halo, $m_{\text {halo }}(r)$, and that of the $\psi$-field. So, integrating Eq. (8) above yields

$\ln \left(r v_{\theta}\right)=\ln \mathcal{J}+\frac{\partial g}{\partial \psi}\left(\psi_{\infty}\right) \psi_{\infty}\left(\psi-\psi_{\infty}\right)$,

where $\mathcal{J}$ would represent the angular momentum per unit mass if the $\psi$-field were not excited. Hereafter, we assume $\frac{\partial g}{\partial \psi}\left(\psi_{\infty}\right) \psi_{\infty}>0$ so that $m^{(\psi)}(r)$ mimics a hidden mass profile ${ }^{1}$. In addition, we adopt the model of a quasi-isothermal spherical dark halo, that is, a mass density profile such that

$\rho_{\text {halo }}(r)=\rho_{\text {halo }}\left(r_{0}\right)\left(\frac{r_{0}}{r}\right)^{2+\varepsilon}$

${ }^{1}$ Since $\psi$ also acts as a stabilizing field (see Mbelek \& Lachièze-Rey 2003), one may expect that it affects the dynamics within the galaxy by lowering the magnitude of the orbital momentum per unit mass at any given radius. This reads $r v_{\theta}<\mathcal{J}$ and consequently $\delta \psi<0$ on account of Eq. (10) and by assuming $\frac{\partial g}{\partial \psi}\left(\psi_{\infty}\right) \psi_{\infty}>0$. Consequently, the consistency with $\psi_{\infty}$ defining the minimum of the potential $U(\psi)$ at $r \rightarrow \infty$ implies that $\mathrm{d} \psi / \mathrm{d} r>0$ for $\delta \psi$ increasing monotonously. 
where $\varepsilon$ is a free parameter which depends on the given galaxy and lies within the range $\left.] 0, \frac{1}{2}\right]$. We show below that the use of an isothermal dark halo together with the possibility of noncircular orbits may explain the apparent discrepancy with the Navarro-Frenk-White (NFW) or Burkert halos assuming circular orbits for the latter. Recently, Bournaud et al. (2003) have proved for the first time from $N$-body simulation that a large extent of dark matter is necessary for the formation of tidal dwarf galaxies. In this study, the authors described the dark matter as a standard isothermal sphere. Hence, although the main concern of the authors is the tidal interaction in the outskirts of galaxies, the hypothesis of an isothermal (or quasi-isothermal sphere) still proves to be worth investigating. For simplicity, we will neglect the contributions of the bulge and the disk (stellar plus gaseous disks) as sources of the $\psi$-field since the dark halo is the major mass component of the galaxies. Thus, one finds that the static spherical solution $\psi$ of Eq. (5) reads

$\psi=\psi_{\infty}-\frac{1}{\varepsilon} \frac{\partial g}{\partial \psi}\left(\psi_{\infty}\right) \psi_{\infty} \frac{G m_{\text {halo }}\left(r_{0}\right)}{r_{0} c^{2}}\left(\frac{r_{0}}{r}\right)^{\varepsilon}$.

Inserting the solution found for $\psi$ in relation (10) above, the tangential velocity expresses in the form

$v_{\theta}=v_{\theta \max } G_{v}(x)$,

where $x=r / r_{0}$ and we have set $v=1 / \varepsilon$ for convenience (see below). The functions $G_{v}$ are defined for $x>0$ as follows

$G_{v}(x)=\frac{1}{x} \exp \left[v\left(1-x^{-1 / v}\right)\right]$.

These functions are positively defined, bounded from above by $G_{\nu}(1)=1$ and satisfy $G_{v}(x)^{k}=G_{k v}\left(x^{k}\right)$. Solving the radial Eq. (9), on account that $\ddot{r}=\mathrm{d} \dot{r} / \mathrm{d} t=\mathrm{d}\left(\frac{1}{2} \dot{r}^{2}\right) / \mathrm{d} r\left(v_{\theta}=v_{\theta}(r)\right.$ and $\dot{r}=\dot{r}(r)$ at radius $r$ ), one finds

$\dot{r}(r)^{2}=\dot{r}\left(r_{0}\right)^{2}+2 \int_{r_{0}}^{r}\left(\frac{v_{\theta}^{2}}{r}-G \frac{m(r)+m^{(\psi)}(r)}{r^{2}}\right) \mathrm{d} r$.

Hence, one expects $m(r)+m^{(\psi)}(r) \simeq v_{\theta}^{2} r / G$ for any good fit to the RC that can be obtained assuming circular orbits, where ${ }^{2}$ $m^{(\psi)}(r)=\left(\frac{\partial g}{\partial \psi}\left(\psi_{\infty}\right) \psi_{\infty}\right)^{2} m_{\text {halo }}\left(r_{0}\right)\left(r / r_{0}\right)^{1-\varepsilon}$. Actually, the departure from circular orbits may help to understand the use of the various dark halo density mass profiles,

$\rho_{\mathrm{DM}}=\rho_{*} \frac{\left(\frac{r}{r_{*}}\right)^{\alpha \beta}}{\left(\frac{r}{r_{*}}\right)^{2}\left(1+\frac{r}{r_{*}}\right)\left[1+\left(\frac{r}{r_{*}}\right)^{\alpha}\right]^{\beta}}$,

that are advocated in the literature to fit the RC of spiral galaxies, where $\alpha=1$ both for NFW and Moore $\left(\beta=\frac{1}{2}\right.$, for the latter) and $\beta=1$ both for NFW and Burkert ( $\alpha=2$, for the latter). Indeed, it follows from Eq. (9) and assuming both conditions $\ddot{r}>0$ and $\mathrm{d}\left(\ddot{r} r^{2} / G\right) / \mathrm{d} r \geq 0$ that the quantity $\ddot{r} r^{2} / G$

${ }^{2}$ The case $\varepsilon=1$, which matches a NFW dark halo density mass profile far beyond the core radius, yields a point mass $m^{(\psi)}=$ $\left(\frac{\partial g}{\partial \psi}\left(\psi_{\infty}\right) \psi_{\infty}\right)^{2} m_{\text {halo }}\left(r_{0}\right)$ that mimics a black hole located at the center of the galaxy.

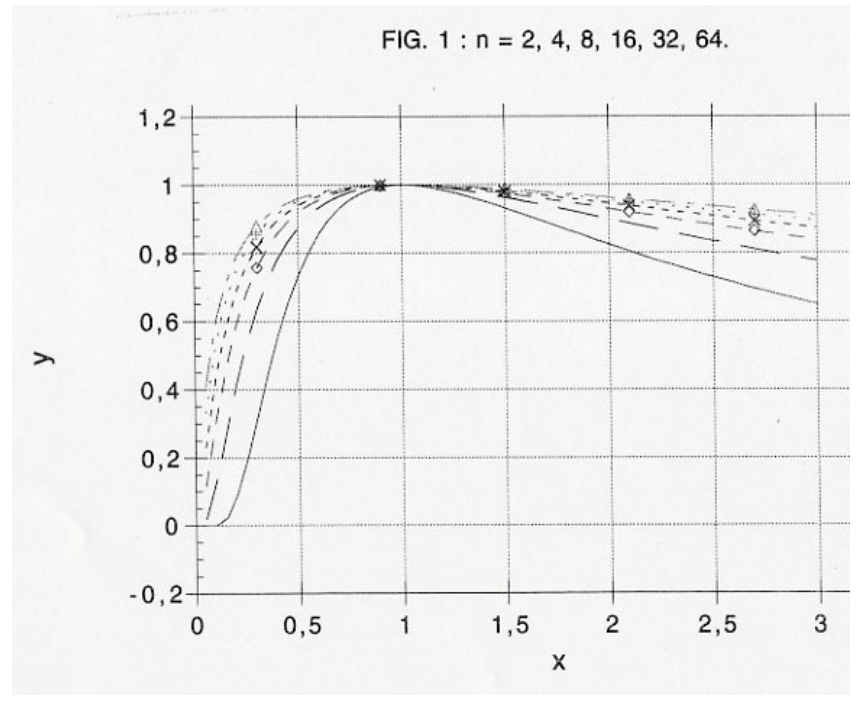

Fig. 1. Generic curves $y=G_{n}(x)$ for $n=2,4,8,16,32$ and 64 (from bottom to top). As one can see, the greater $n$, the steeper is the curve below $x=0.5$ and the flater it is beyond.

behaves like an additional contribution which mimics a mass density profile as if the orbits were circular (Mbelek 1997). Further, $m_{\mathrm{eff}}(r)=m(r)+m^{(\psi)}(r)+G^{-1} \ddot{r} r^{2}$ will define an effective mass profile consistent with relation (16) in the range $r_{\text {min }} \leq r \leq r_{\max }$ such that $r_{\text {min }}=\left(\rho_{\text {halo }}\left(r_{*}\right) / \rho_{*}\right)^{1 / \alpha} r_{*}$ and $r_{\text {max }}=\left(\rho_{*} / \rho_{\text {halo }}\left(r_{*}\right)\right) r_{*} ; \rho_{\text {halo }}\left(r_{*}\right)<\rho_{*}$.

\section{Results}

In the following, we limit our study to those RC for which the radial velocity, $\dot{r}$, may be neglected with respect to the tangential velocity, $v_{\theta}$, in the whole range $r_{\min } \leq r \leq r_{\max }$ where we assume that the dark halo mass density dominates the baryonic mass density. Furthermore, the fits to the individual RC show that $v$ is actually an integer $n \geq 2$. This is consistent with our former work (Mbelek 1998) in the sense that combining the solution found for $\psi$ with relation (11) allows us to express the right-hand side of Eq. (3) as the derivative of an effective power-law potential which varies as $\left(\psi-\psi_{\infty}\right)^{2(n+1)}$. As can be seen in Fig. 1, the greater the $n$, the steeper is the curve $y=v_{\theta} / v_{\theta \max }$ versus $x$ for $x<1$ and the flater it is for $x>1$. Hence, the steepest is a $\mathrm{RC}$ below the turnover radius, and it should be flatest beyond. Figure 2 shows some fits to individual rotation curves from the samples of Rubin et al. (1980, 1985), van Albada et al. (1985), Lake et al. (1990). This is achieved by using the least-squares fit to search the parameters $a$ and $b=\ln J$ that yield the maximum square, $R^{2}$, of the correlation coefficient for the relation $\ln \left(r v_{\theta}\right)=a r^{-1 / n}+b$. Based on the study of a hundred spirals, $a$ is always negative, in agreement with $\delta \psi<0$ whereas $b$ is always positive. In addition, one gets approximately the following statistics: $n=5$ for $20 \%$ of the spirals, $3 \leq n \leq 6$ for almost a half of them and $3 \leq n \leq 12$ for $80 \%$ of them.

Figure 3 shows that there is a strong correlation between both coefficients $a$ and $b$. Although displayed here for sixteen spirals only, this remains true for the whole sample of a 

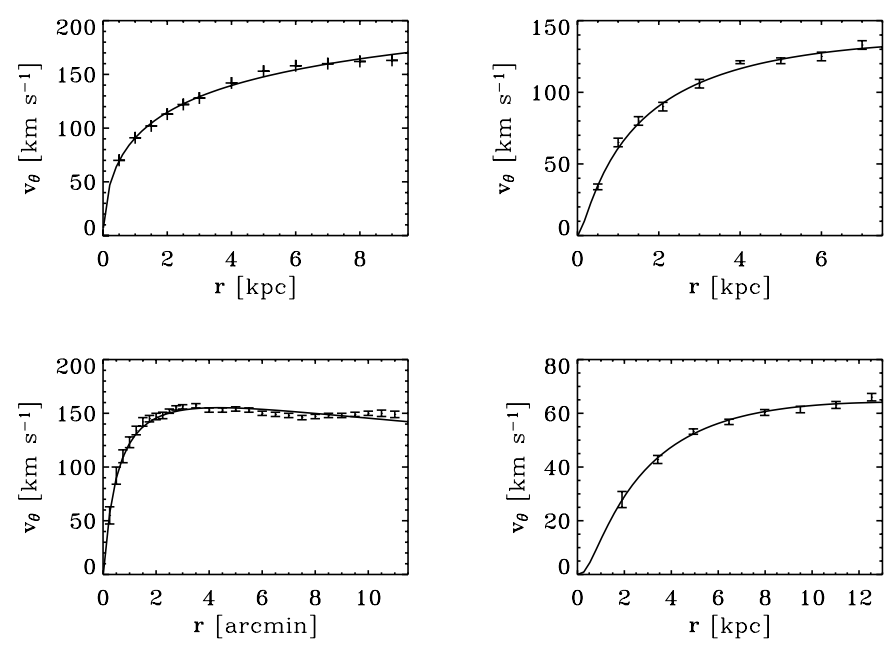

Fig. 2. Rotation curve fits (from top to bottom) for NGC 4062 (Rubin et al. 1980), NGC 1035 (Rubin et al. 1985), NGC 3198 (van Albada et al. 1985,1 arcmin $=2.68 \mathrm{kpc}$ ) and DDO 170 (Lake et al. 1990); caption: the crosses or error bars are the observed data and the solid line is the best fit of the RC.

hundred spirals we have studied. Expressing $r$ in $k p c$ for all galaxies, one finds ${ }^{3} b=|a|+4.25$ with $R=0.995$. More generally, there are strong linear correlations between the integer $n$ and the coefficients $a$ and $b$. Now, the theoretical relations between the fitting parameters $(a, b)$ and the physical ones $\left(r_{0}, v_{\theta \max }\right)$ are given by $r_{0}=(|a| / n)^{n}$ and $b=n+\ln \left(r_{0} v_{\theta \max }\right)$. From the latter relation, it is clear that the correlation between $b$ and $n$ is obtained by the logarithm which alleviates the effect of the scatter in the quantity $r_{0} v_{\theta \max }$. As for the correlation between $a$ and $n$, it suggests a relation of the kind $r_{0}=\alpha\left(1+\frac{\beta}{n}\right)^{n}$, where $\alpha \simeq 1 \mathrm{kpc}$ and $\beta$ is a dimensionless positive constant almost independent of the given spiral galaxy. Hence, if a natural theoretical explanation could be found for the relation between $r_{0}$ and $n$, only one free parameter, namely $n$, would be needed to fit the RC. Since the latter is just an integer, it is tempting to believe that this feature may reveal the nature of quantification underlying the galactic dark halo mass distributions.

\section{Conclusion}

In this paper, we have shown based on a simple mass model that a real scalar field minimally coupled to gravity and with matter as its source may reproduce the RC of spiral galaxies. This is achieved even if the scalar field does not contribute significantly to the galactic dark matter mass. Moreover, such a scalar field may also act as a stabilizing field. The new correlations that have been found open the possibility to fit the RC with only one single free parameter which is an integer. Thus, it seems possible that long-range scalar fields minimally coupled to gravity play a significant role not only at the cosmological scale (Mbelek \& Lachièze-Rey 2003; del Campo \& Salgado 2003; Caresia et al. 2004) but also within galaxies.

Acknowledgements. I thank the anonymous referee for useful comments and suggestions that helped to improve the manuscript.

\footnotetext{
${ }^{3}$ For the whole sample, $H_{0}$ is in the range $50-75 \mathrm{~km} \mathrm{~s}^{-1} \mathrm{Mpc}^{-1}$.
}

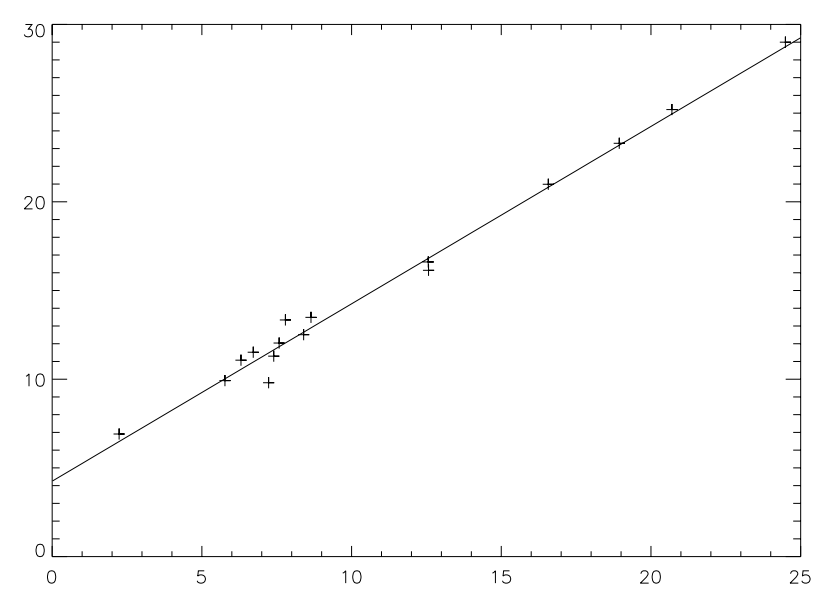

Fig. 3. Plot of the coefficient $b$ versus the coefficient $|a|$ for the galaxies NGC 4062 and UGC 3691 from the sample of Rubin et al. (1980), NGC 4419, 1035, 1325, 2742, 3067, 3593, 2639, 4378, 4448, 7606 and UGC 12810 from the sample of Rubin et al. (1985), NGC 3198 (van Albada et al. 1985), DDO 170 (Lake et al. 1990) and NGC 4051 (Kaneko et al. 1997). One can see the strong linear correlation between $|a|$ and $b$; the crosses are the data from the fits to individual galaxies and the solid line is the best fit to the whole sample of a hundred galaxies which expresses as $b=|a|+4.25$ with correlation coeffient $R=0.995$.

\section{References}

van Albada, T. S., Bahcall, J. N., Begeman, K., \& Sancisi, R. 1985, ApJ, 295, 305

Arbey, A., Lesgourgues, J., \& Salati, P. 2001a, Phys. Rev. D, 64, 123528

Arbey, A., Lesgourgues, J., \& Salati, P. 2001b, Phys. Rev. D, 65, 083514

Brans, C., \& Dicke, R. H. 1961, Phys. Rev., 124, 925, Appendix

Bournaud, F., Duc, P.-A., \& Masset, F. 2003, A\&A, 411, L469

del Campo, S., \& Salgado, P. 2003, Class. Quant. Grav., 20, 4331

Caresia, P., Matarrese, S., \& Moscardini, L. 2004, ApJ, 605, 21

Ciufolini, I., \& Wheeler, J. A. 1995, Gravitation and Inertia (Princeton: Princeton University Press), 29

Guzman, F. S., \& Matos, T. 2000a, Class. Quant. Grav., 17, L9

Guzman, F. S., \& Matos, T. 2000b, Phys. Rev. D, 62, 061301

Kaneko, N., Aoki, K., Kosugi, G., et al. 1997, AJ, 114, 94

Lake, G. L., Schommer, R. A., \& van Gorkom, J. H. 1990, AJ, 99, 547

Mbelek, J. P. 1997, Proc. Eighth Rencontres de Blois Neutrinos, Dark Matter and the Universe, ed. T. Stolarczyk, J. Tran Thanh Van, \& F. Vannucci, 391

Mbelek, J. P. 1998, Acta Cosmologica, XXIV-1, 127, and [gr-qc/0402084]

Mbelek, J. P., \& Lachièze-Rey, M. 2003, A\&A, 397, 803

Narlikar, J. V., \& Padmanabhan, T. 1986, Gravity, Gauge Theory and Quantum Cosmology (Dordrecht: D. Reidel Publishing Company), 70

Rubin, V. C., Ford, W. K., Jr., \& Thonnard, N. 1980, ApJ, 238, 471

Rubin, V. C., Burstein, D., Ford W. K., Jr., \& Thonnard, N. 1985, ApJ, 289,81

Straumann, N. 2000, in Gauge Theory and Gravitation, Zuoz Proceedings, http://ltpth.web.psi.ch/zuoz/zuoz2000/ zuoz2000proc.htm, Sect. 2.1

Ureña-Lòpez, L. A., \& Liddle, A. R. 2002, Phys. Rev. D, 66, 083005 Wesson, P. S., \& Ponce de Leon, J. 1995, A\&A, 294, 1 